\title{
Extending the Root-Locus Method to Fractional-Order Systems
}

\author{
Farshad Merrikh-Bayat ${ }^{1}$ and Mahdi Afshar ${ }^{2}$ \\ ${ }^{1}$ Department of Electrical Engineering, Zanjan University, Zanjan, Iran \\ ${ }^{2}$ Department of Mathematics, Zanjan Azad University, Zanjan, Iran \\ Correspondence should be addressed to Farshad Merrikh-Bayat, f.bayat@gmail.com
}

Received 16 September 2007; Revised 11 March 2008; Accepted 14 May 2008

Recommended by Alberto Tesi

The well-known root-locus method is developed for special subset of linear time-invariant systems known as fractional-order systems. Transfer functions of these systems are rational functions with polynomials of rational powers of the Laplace variable $s$. Such systems are defined on a Riemann surface because of their multivalued nature. A set of rules for plotting the root loci on the first Riemann sheet is presented. The important features of the classical root-locus method such as asymptotes, roots condition on the real axis, and breakaway points are extended to fractional case. It is also shown that the proposed method can assess the closed-loop stability of fractional-order systems in the presence of a varying gain in the loop. Three illustrative examples are presented to confirm the effectiveness of the proposed algorithm.

Copyright (C) 2008 F. Merrikh-Bayat and M. Afshar. This is an open access article distributed under the Creative Commons Attribution License, which permits unrestricted use, distribution, and reproduction in any medium, provided the original work is properly cited.

\section{Introduction}

The root-locus method of Evans is one of the most popular and powerful tools for both analysis and design of single-input single-output (SISO) linear time-invariant (LTI) systems. There are two main application areas for this method [1] as follows. (1) Stability: to obtain sufficient conditions on a real parameter $k$ under which the closed-loop system in Figure 1 remains stable. (2) Design: the root-locus method offers an efficient tool for design of lead-lag compensators. There have been further advances to the root-locus method since its origin in 1948. Krall [2,3] developed the method for delayed systems, Bahar and Fitzwater [4] studied the problem from the numerical point of view and finally, Byrnes et al. [5] presented the rootlocus method for distributed parameter systems.

For typical systems, there are several easy-to-use rules for plotting the root loci that do not generally suffice to determine it uniquely [1]. These rules serve only as hints and often the 


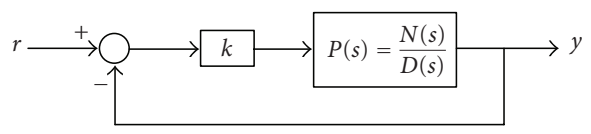

Figure 1: Standard closed-loop system.

intuitive insight of a control engineer is needed for completing the root-locus plot. The rootlocus method will apparently become more difficult to apply as the system's order becomes higher [5].

In recent years, there has been an increasing attention to fractional-order systems. These systems are of interest for both modelling and controller design purposes. In the fields of continuous-time modelling, fractional derivatives have proved useful in linear viscoelasticity, acoustics, rheology, polymeric chemistry, biophysics,... [6, 7]. In general, fractional-order systems are useful to model various stable physical phenomena (commonly diffusive systems) with anomalous decay.

An interesting study of fractional differential systems appeared in [8] using a stochastic framework. The idea of fractional powers is also used for identification purposes. Tsao et al. [9] and Poinot and Trigeassou [10] clarify the identification method when the members of model set are of fractional order. Two applications of such identifications can be found in [11, 12]. Fractional-order systems are also used in control field. Podlubny [13] and Valério and Sá da Costa [14] discussed methods of designing $\mathrm{PI}^{\lambda} \mathrm{D}^{\mu}$ controllers, Raynaud and Zergaïnoh [15] studied fractional-order lead-lag compensators, and Oustaloup et al. [16, 17] introduced the so-called CRONE controllers.

In this paper, the systems under consideration are described by rational transfer functions and the powers of the Laplace variable, $s$, are limited to rational numbers. Such systems lend themselves well to some algebraic tools [18, 19]. Practical examples of such systems can be found in $[11,12,19]$. The problem of plotting the root loci for these systems is treated in this paper. Unlike [5] that deals with infinite-dimensional systems, in the problem we are going to solve, the systems are assumed to be of finite dimension and this makes the problem simple enough to deal with analytically.

The proposed method can be used to examine whether a given closed-loop system, as shown in Figure 1, remains stable for large $k^{\prime}$ s or not, where $P(s)$ is a fractional-order transfer function. In [20], a generalization of the Routh-Hurwitz criterion for fractional-order systems is presented. However, this method can deal with the stability problem for such systems but it is a very complicated algorithm.

The rest of this paper is arranged as follows. Section 2 provides some basic definitions and notations together with the problem statement. In Section 3, the rules for plotting the root loci in fractional case are presented. Three illustrative examples are presented in Section 4. Finally, some conclusions end the paper.

\section{Notation}

Blackboard capitals denote sets and spaces: especially $\mathbb{N}$ the natural numbers (without zero), $\mathbb{R}$ the real numbers, $\mathbb{Q}$ the rational numbers, $\mathbb{Z}$ the integer numbers, and $\mathbb{C}$ the complex numbers. The symbol $\lfloor x\rfloor$, where $x \in \mathbb{R}$, denotes the biggest integer that is less than or equal to $x$. 


\section{Problem statement and preliminaries}

Before introducing the main problem, some basic definitions and notations are provided. It is assumed that the reader is familiar with the concepts of "Riemann surface," "Riemann sheet," "branch point," and "branch cut" (see, e.g., [21], or [22] for deeper analysis).

Definition 2.1. The function $Q(s)=a_{1} s^{q_{1}}+a_{2} s^{q_{2}}+\cdots+a_{n} s^{q_{n}}$ is a fractional-order polynomial, if and only if $q_{i} \in \mathbb{Q}^{+} \cup\{0\}, a_{i} \in \mathbb{R}$, for $i=1, \ldots, n$.

Definition 2.2. Consider the fractional-order polynomial

$$
Q(s)=a_{1} s^{\alpha_{1} / \beta_{1}}+a_{2} s^{\alpha_{2} / \beta_{2}}+\cdots+a_{n} s^{\alpha_{n} / \beta_{n}}, \quad a_{i} \in \mathbb{R}, \alpha_{i} \in \mathbb{N} \cup\{0\}, \beta_{i} \in \mathbb{N},
$$

where $\alpha_{i}, \beta_{i}$ are relatively prime for $i=1, \ldots, n$. (If for some $i, \alpha_{i}=0$ then by definition $\beta_{i}=1$.) Let $\lambda$ be the least common multiple (lcm) of $\beta_{1}, \beta_{2}, \ldots, \beta_{n}$ denoted as $\lambda=\operatorname{lcm}\left\{\beta_{1}, \beta_{2}, \ldots, \beta_{n}\right\}$. Then $Q(s)$ can be written as

$$
Q(s)=a_{1}\left(s^{1 / \lambda}\right)^{\lambda_{1}}+a_{2}\left(s^{1 / \lambda}\right)^{\lambda_{2}}+\cdots+a_{n}\left(s^{1 / \lambda}\right)^{\lambda_{n}} .
$$

Now the fractional degree (fdeg) of $Q(s)$ is defined as

$$
\operatorname{fdeg}\{Q(s)\}=\max \left\{\lambda_{1}, \lambda_{2}, \ldots, \lambda_{n}\right\} .
$$

The function $Q$ as defined in (2.2) is a multivalued relation of $s$ the domain of definition for which is a Riemann surface with $\lambda$ Riemann sheets where the origin is a branch point [21]. In this paper, the branch cut is assumed at $\mathbb{R}^{-}$and the first Riemann sheet is denoted by $P$ and defined as

$$
D:=\left\{r e^{i \theta} \mid r>0,-\pi<\theta \leq \pi\right\}
$$

Note that each Riemann sheet has only one edge at branch cut. The following proposition gives the roots number of $Q(s)=0$.

Proposition 2.3. Let $Q(s)$ be a fractional-order polynomial with $\operatorname{fdeg}\{Q(s)\}=n$. Then the equation $Q(s)=0$ has exactly $n$ roots on the Riemann surface.

Proof. Consider

$$
Q(s)=a_{1}\left(s^{1 / v}\right)^{n}+a_{2}\left(s^{1 / v}\right)^{n-1}+\cdots+a_{n}\left(s^{1 / v}\right)^{1}+a_{n+1}
$$

for an appropriate $v \in \mathbb{N}$. Assuming $w:=s^{1 / v}$, we have

$$
\widetilde{Q}(w)=a_{1} w^{n}+a_{1} w^{n-1}+\cdots+a_{n} w+a_{n+1} .
$$

The fundamental theorem of algebra gives $n$ roots for $\widetilde{Q}(w)=0$, say $w_{1}, w_{2}, \ldots, w_{n}$. Consequently, $Q(s)=0$ has $n$ roots at $s_{1}=w_{1}^{v}, s_{2}=w_{2}^{v}, \ldots, s_{n}=w_{n}^{v}$. 
Definition 2.4. The fractional-order polynomial $Q(s)=a_{0} s^{n / v}+a_{1} s^{(n-1) / v}+\cdots+a_{n-1} s^{1 / v}+a_{n}$ is minimal if $\operatorname{fdeg}\{\mathrm{Q}(\mathrm{s})\}=n$.

Now consider the standard closed-loop system in Figure 1 where the transfer function of plant is given by

$$
P(s)=\frac{N(s)}{D(s)}=\frac{s^{m / v}+b_{1} s^{(m-1) / v}+\cdots+b_{m-1} s^{1 / v}+b_{m}}{s^{n / v}+a_{1} s^{(n-1) / v}+\cdots+a_{n-1} s^{1 / v}+a_{n}}, \quad(v>1),
$$

and $k$ is assumed to be a positive real constant. Note that the domain of definition of $P(s)$ is a Riemann surface with $v$ Riemann sheets [21].

Definition 2.5. With the above notations, $P(s)$ is called strictly proper for $n>m$, proper for $n \geq m$, nonproper for $n<m$, and biproper for $n=m$.

Definition 2.6. The roots of the equations $N(s)=0$ and $D(s)=0$ on $D$ are called open-loop zeros and open-loop poles, respectively.

It is a fact that when a minimal fractional-order polynomial is represented in a nonminimal form, the number of its zeros is increased but the location and the order of zeros on $p$ remain unchanged. For example, consider the fractional-order polynomials $f(s)=s^{1 / 2}-1$ (minimal) and $g(s)=s^{2 / 4}-1$ (nonminimal). The equation $f(s)=0$ has only one root at $s=e^{i 0}$ (on the first Riemann sheet) while $g(s)=0$ has a root at $s=e^{i 0}$ (on the first Riemann sheet), and another root at $s=e^{i 4 \pi}$ (on the third Riemann sheet), although $f(s)$ and $g(s)$ have different number of zeros but the location and the order of their zero on $P$ are identical. It concludes that Definition 2.6 is not ambiguous; representing $N(s)$ and $D(s)$ in a nonminimal form will not affect the open-loop poles and zeros.

Note that $s=0$ is not a pole of $P(s)$ even if $D(0)=0$. The following definition deals with the singularities at the origin.

Definition 2.7. The point $s=0$ is defined to be a pole of order $r$ of $P(s)$ (as defined in (2.7)) if the point $w=0$ is a pole of order $r$ of $\widetilde{P}(w):=\left.P(s)\right|_{w=s^{1 / v}}$.

The characteristic equation of the closed-loop system shown in Figure 1 is

$$
\begin{aligned}
\Delta(s) & =D(s)+k N(s) \\
& =s^{n / v}+a_{1} s^{(n-1) / v}+\cdots+a_{n-1} s^{1 / v}+a_{n}+k\left[s^{m / v}+b_{1} s^{(m-1) / v}+\cdots+b_{m-1} s^{1 / v}+b_{m}\right]=0 .
\end{aligned}
$$

It is desired to address the generalized root-locus problem that is to plot the root loci of (2.8) on $p$ when $k$ varies. The reason for concerning about the first Riemann sheet is that the timedomain behavior and stability properties of the closed-loop system are determined only by those roots of the characteristic equation that lie on the first Riemann sheet $[19,23]$. Note that a system with characteristic equation (2.8) is stable (in the sense of bounded-input boundedoutput) if and only if it has no roots in the closed right half plane (CRHP) of $D$ [24, 25]. 


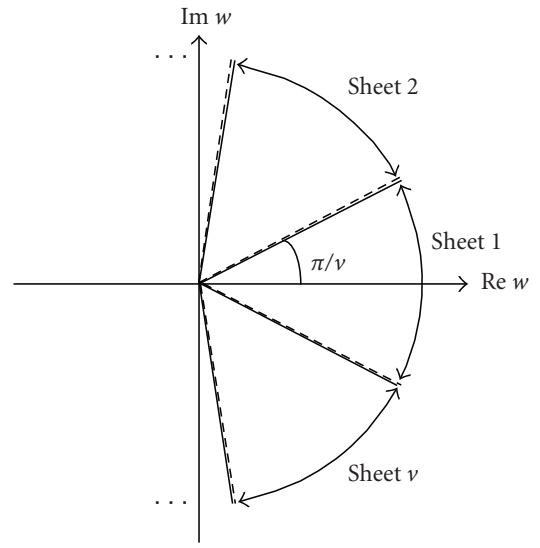

Figure 2: The correspondence between $w$-plane and $s$ Riemann sheets.

In this paper, we restrict ourselves to the following conditions.

(i) The transfer function of the plant is strictly proper. Final results can easily be extended for nonproper systems but such an extension to biproper transfer functions is complicated. This is similar to the difficulty that occurs when the root-locus plot for an integer-order system with biproper transfer function is involved [26].

(ii) Parameter $k$ is a positive real number. The results can easily be extended for negative real $k^{\prime}$ s.

(iii) Both $N(s)$ and $D(s)$ are monic fractional-order polynomials. This does not lose the generality and simplifies the notations.

(iv) $N(s)$ and $D(s)$ have no common roots. Otherwise, the characteristic equation will have $\operatorname{root}(\mathrm{s})$ that does (do) not vary by changing $k$.

In the rest of this paper, the single-valued function obtained by replacing every $s^{1 / v}$ in (2.8) with $w$ is denoted by $\widetilde{\Delta}(w)$, that is,

$$
\tilde{\Delta}(w)=w^{n}+a_{1} w^{n-1}+\cdots+a_{n-1} w+a_{n}+k\left[w^{m}+b_{1} w^{m-1}+\cdots+b_{m-1} w+b_{m}\right]=0 .
$$

We do the same for other multivalued relations.

\section{Root loci in fractional case}

\subsection{Properties of the root loci in fractional case}

The root-locus plot of $\widetilde{\Delta}(w)=0$ provides a very good insight to the root-locus plot of $\Delta(s)$. Figure 2 shows the relationship between $w$-plane and sheets of the $s$ Riemann surface. In this figure, the sector $-\pi / v<\arg w \leq \pi / v$ corresponds to $D$. In the following, some important features of the root loci of $\Delta(s)$ are presented. 


\subsubsection{Symmetry with respect to real axis}

Considering the fact that the root loci of $\widetilde{\Delta}(w)=0$ is symmetric with respect to real axis, it is concluded that the roots loci of $\Delta(s)$ on $D$ are also symmetric with respect to real axis. Note that, in general, this explanation is not correct for the root loci on other Riemann sheets.

\subsubsection{Number of branches}

A branch by definition is the loci of a single root of the characteristic equation when $k$ varies from zero to infinity. In classical case, the root-locus branches start from open-loop poles and terminate at zeros (finite zeros or zeros at infinity) [1]. In fractional case, it is concluded from Proposition 2.3 that the characteristic equation (2.8) has $n$ roots distributed on $v$ Riemann sheets. Considering the root-locus plot of $\widetilde{\Delta}(w)$ in $w$-plane, it is obvious that not all root-locus branches on necessarily start from open-loop poles and terminate at open-loop zeros. In fact, a branch may cross the branch cut and enter to another Riemann sheet.

There is another point that should be noted here. Clearly, $r$ branches start from the openloop pole $s_{0} \in D$ which is of order $r$. When $s_{0} \notin \mathbb{R}^{-}$, all these branches are on $D$ for $k \rightarrow 0^{+}$. Otherwise, they belong to different Riemann sheets. One important case is due to the poles at the origin. If $s_{1}=0$ is a pole of order $r$ of (2.7), then $r$ branches start it, which are not necessarily on for $k \rightarrow 0^{+}$. In order to find the number of branches that start from $s_{1}$ and are on $p$ for $k \rightarrow 0^{+}$, let $p$ and $q$ stand for the number of positive real open-loop poles and zeros, respectively. Then according to the angle condition, the angle of departure from the pole at the origin is obtained as

$$
\phi_{h}=\frac{2 h+1-p+q}{r} v \pi, \quad h=\left\lfloor\frac{p-q-(n / v)-1}{2}\right\rfloor+1, \ldots,\left\lfloor\frac{p-q+(n / v)-1}{2}\right\rfloor .
$$

As a result, if (2.7) has a pole of order $r$ at the origin, then $\lfloor(p-q+(n / v)-1) / 2\rfloor-\lfloor(p-q-$ $(n / v)-1) / 2$ J branches $($ on $P$ ) start from $s=0$ the angle of departure of which is calculated from (3.1).

\subsubsection{Roots conditions on the real axis}

In the classical root-locus algorithm, any point on the real axis, the total number of real poles and zeros to the right of which is odd, lies on a root locus. Clearly, the line segments lying on the positive real axis of $w$-plane are mapped to the line segments lying on the positive real axis of $p$. So, according to the root-locus plot in $w$-plane, any point on the positive real axis of $p$, the total number of real poles and zeros to the right of which is odd, lies on a root locus. But for $P(s)$ given in (2.7), no line segment on $\mathbb{R}^{-}$can belong to the root locus. The reason is as follows. If such a line exists then it should necessarily lie on the ray $r e^{i \pi / v}(r>0)$ in $w$-plane. It is a well-known classical result that the semiinfinite line $r e^{i \pi / v}(r>\sqrt[v]{x})$, which is a root loci branch of a system with transfer function $\widetilde{P}(w)=\left(1 /\left(w^{v}+x\right)\right)\left(x \in \mathbb{R}^{+}\right)$, is the only object in $w$ plane that can lie on this ray. But $\widetilde{P}(w)$ corresponds to $P(s)=1 /\left(\left(s^{1 / v}\right)^{v}+x\right)=1 /(s+x)$ which is not multivalued. Consequently, the root-locus plot of the multivalued transfer function $P(s)$ can never have a branch at $\mathbb{R}^{-}$. 


\subsubsection{Asymptotes and their directions}

Asymptotes are very important in drawing a root-locus plot as they exhibit directions of the branches for large $k^{\prime}$ s. The asymptotes of the root-locus plot in integer case are first studied in [21]. Another explanation with more details can be found in [27]. The approach used in [21,27] cannot directly be applied for fractional case. Here we develop an alternative approach to find the asymptotes to the root-locus curves of a fractional-order transfer function. The following theorem is the main result of this paper because it can be used to examine the closed-loop stability for large gains.

Theorem 3.1. Asymptotes of the root-locus plot are straight lines all passing through the origin and their directions are given by

$$
\varphi_{h}=\frac{(2 h+1) v}{n-m} 180^{\circ}, \quad h=\left\lfloor\frac{m-n-v}{2 v}\right\rfloor+1, \ldots,\left\lfloor\frac{m-n-v}{2 v}\right\rfloor+n-m .
$$

Proof. See Appendix.

Note that in Theorem 3.1, $h$ may belong to any sequence of $n-m$ successive integer numbers but the sequence we have used makes a relevant correspondence between asymptotes and Riemann sheets. This sequence guarantees that $-180^{\circ}<\varphi_{h}<360^{\circ} v-180^{\circ}$. Note also that for $h=\lfloor(m-n-v) / 2 v\rfloor+1, \ldots,\lfloor(n-m-v) / 2 v\rfloor$ the resulting asymptotes lie on $p$. In fractional case, however, all asymptotes pass through the origin. Since the open-loop system is assumed to be strictly proper, the root-locus plot will always have at least one asymptote.

Remark 3.2. In integer case, if the negative real axis is an asymptote for the root-locus plot, then it is the asymptote for one and only one of the branches. Moreover, the corresponding branch of the root-locus plot coincides with the asymptote. In the fractional case, however, the negative real axis can be an asymptote line for more than one root and it is not superposed on any branch. As a matter of fact, according to (3.2) the negative real axis is the asymptote for all infinite roots when $n-m=v$.

\subsubsection{Breakaway and break-in points}

The breakaway and break-in points on a root-locus plot are points where two or more branches intersect and then go apart. If $s_{0}$ is a breakaway (break-in) point then it necessarily satisfies both $\Delta\left(s_{0}\right)=0$ and $\mathrm{d} \Delta\left(s_{0}\right) / \mathrm{d} s=0$ for some $s_{0}$ on $p$. These two equations imply that $D\left(s_{0}\right)+k N\left(s_{0}\right)=0$ and $\mathrm{d} D\left(s_{0}\right) / \mathrm{d} s+k \mathrm{~d} N\left(s_{0}\right) / \mathrm{d} s=0$ or equivalently, $N\left(s_{0}\right) \mathrm{d} D\left(s_{0}\right) / \mathrm{d} s-$ $D\left(s_{0}\right) \mathrm{d} N\left(s_{0}\right) / \mathrm{d} s=0$. The latter equation can be interpreted in terms of the open-loop transfer function, $P(s)$, as $\mathrm{d} P\left(s_{0}\right) / \mathrm{d} s=0$. So, every breakaway (break-in) point must satisfy the equation

$$
\frac{\mathrm{d} P(s)}{\mathrm{d} s}=0,
$$

on $D$ (see, e.g., [21] for differentiation of multivalued relations). The roots of (3.3) are breakaway (break-in) points if the corresponding $k$ 's are positive real numbers. Equation (3.3) can also be interpreted in terms of $\widetilde{P}(w)$ as $\mathrm{d} \widetilde{P}(w) / \mathrm{d} w=0$. 


\subsection{Comprehensive algorithm}

The following is a summarization of the general rules for constructing the root loci in fractional case.

(1) Locate the open-loop poles and zeros.

(2) Determine the order of the pole at the origin (if any) and calculate the angle of departure using (3.1).

(3) Determine the root loci on the positive real axis. Note that no line segment on the negative real axis can belong to the root loci.

(4) Determine the directions of asymptotes from (3.2).

(5) Find the breakaway and break-in points (in any) from (3.3).

(6) Complete the root-locus plot.

\section{Examples}

Example 4.1. Consider the closed-loop system in Figure 1 with

$$
P(s)=\frac{s^{1 / 2}-1}{s^{2}-3 s^{3 / 2}-2 s+2 s^{1 / 2}+12} .
$$

The open-loop poles of this system are located at $s=4 e^{i 0}$ and $s=9 e^{i 0}$, and there is an openloop zero located at $s=1 e^{i 0}$. The line segments $0 \leq \mathfrak{R}\{s\} \leq 1$ and $4 \leq \mathfrak{R}\{s\} \leq 9$ belong to the root loci because the total number of poles and zeros to the right of any point on them is an odd number. The directions of the asymptotes (on the first Riemann sheet) are $\varphi_{-1}=-120^{\circ}$ and $\varphi_{0}=120^{\circ}$. The roots of the equation $\mathrm{d} \widetilde{P}(w) / \mathrm{d} w$ are $2.4820,-0.9599$, and $0.9056 \pm i 1.0671$. The only feasible solution is $w=2.4820$ which corresponds to $s=6.1603 e^{i 0}$. Figure 3 shows the root-locus plot of this system.

Example 4.2. Consider the closed-loop system in Figure 1 with

$$
P(s)=\frac{s^{1 / 2}-\sqrt{2}}{s^{2}\left(s^{1 / 2}-1\right)^{3}} .
$$

This system has an open-loop zero at $s=2 e^{i 0}$ and an open-loop pole of order three at $s=1 e^{i 0}$. There is also a pole of order four at the origin. The line segment $1 \leq \mathfrak{R}\{s\} \leq 2$ on the positive real axis belongs to the root loci. According to (3.1), the angles of departure from the pole at the origin are $\phi_{0}=-\pi / 2$ and $\phi_{1}=\pi / 2$. Letting $n=7, m=1$, and $v=2$, it is concluded from (3.2) that the directions of the asymptotes on $D$ are $\varphi_{-1}=-60^{\circ}, \varphi_{0}=60^{\circ}$, and $\varphi_{1}=180^{\circ}$. Figure 4 shows the root-locus plot of this system.

Example 4.3. According to [28], the fractional-order model of a heating furnace is given by

$$
P(s)=\frac{1}{14994 s^{1.131}+6009.5 s^{0.97}+1.69},
$$




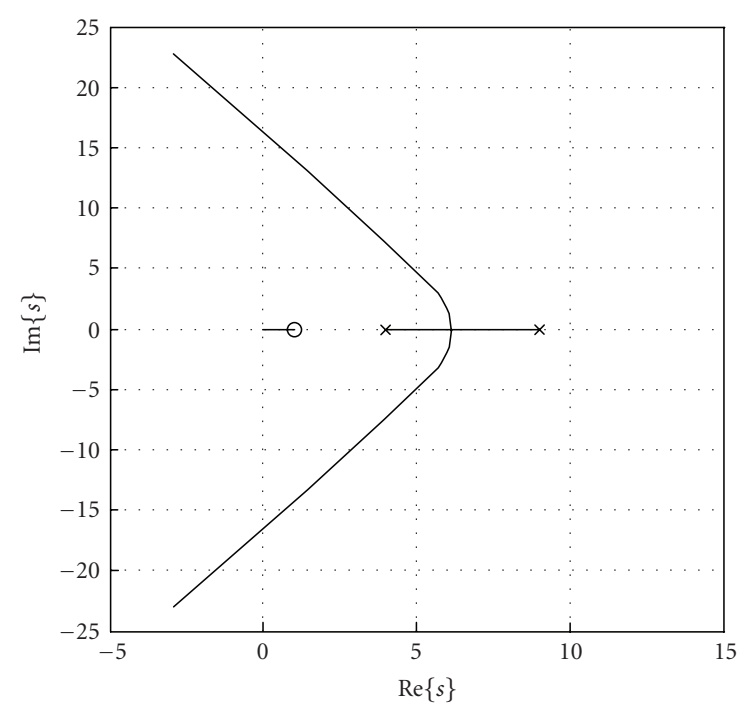

Figure 3: Root-locus plot of Example 4.1.

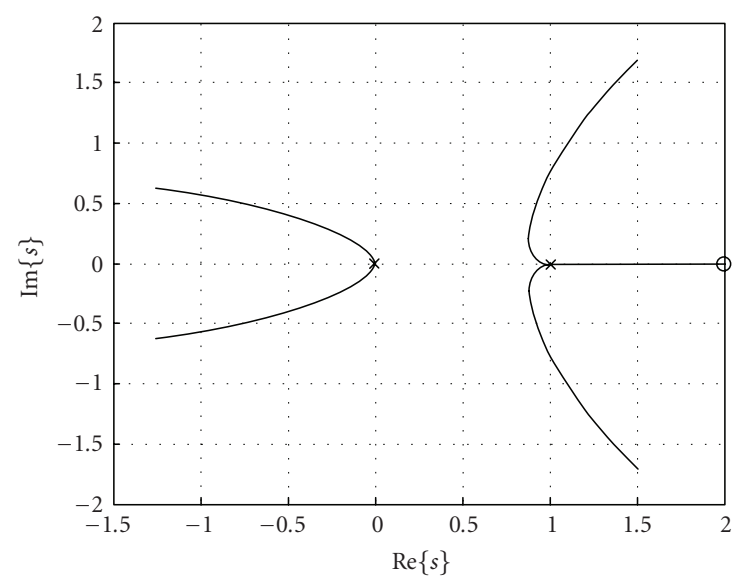

Figure 4: Root-locus plot of Example 4.2.

which compared to (2.7) results in $n=131, m=0$, and $v=100$. Assuming $P(s)$ in the connection of Figure 1, the root-locus plot has two asymptotes on the directions of which are $\varphi_{-1} \approx-137.4^{\circ}$ and $\varphi_{0} \approx 137.4^{\circ}$, thanks to (3.2). Since none of these asymptotes lie on the CRHP of $D$ and $P(s)$ has no zeros, one can choose $k$ arbitrarily large to arrive at a closedloop system with the ability of tracking the command input. Figure 5 shows the closed-loop system response for $k=5,10,20$ together with the open-loop system response when the system is subjected to a unit step. As it is expected, the closed-loop system is stable and its bandwidth is increased by increasing $k$. Podlubny [29] proposed the fractional-order model

$$
P(s)=\frac{1}{0.7943 s^{2.5708}+5.2385 s^{0.8372}+1.5560},
$$




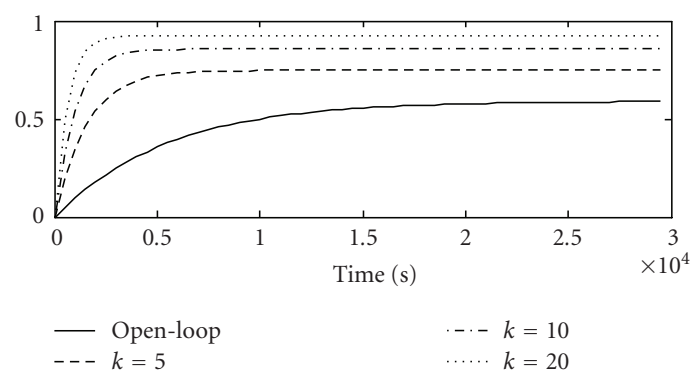

Figure 5: Step responses corresponding to Example 4.3.

for another heating furnace. This transfer function can be represented in the equivalent form

$$
P(s)=\frac{1}{0.7943 s^{6427 / 2500}+5.2385 s^{2093 / 2500}+1.5560},
$$

which corresponds to $n=6427, m=0$, and $v=2500$. The root-locus plot of this system has two unstable infinite branches the directions of which are $\varphi_{-1} \approx-70.02^{\circ}$ and $\varphi_{0} \approx 70.02^{\circ}$. Hence, it is not possible to control the system by means of a simple proportional action. One possible approach is to use more complex structures such as lead-lag compensators.

\section{Conclusion}

In this paper, an approach for constructing the root-locus plot for fractional-order systems is developed. Important features of the root loci are studied and a comprehensive algorithm is presented. Although the rules for plotting the root loci in fractional case are somehow similar to those available in integer case, there are also some major differences. For example, it is shown that in fractional case no line segment on $\mathbb{R}^{-}$can belong to the root loci. It is also shown that the asymptotes of all infinite branches of the root-locus plot pass through the origin. As another difference, the well-known Routh-Hurwitz stability test cannot be used to find the points at which the root-locus plot intersects with the imaginary axis. Three illustrative examples are presented to confirm the effectiveness of the proposed algorithm.

\section{Appendix}

The following is a proof for Theorem 3.1. Consider the general form of the characteristic equation as follows:

$$
1+k \frac{s^{m / v}+b_{1} s^{(m-1) / v}+\cdots+b_{m}}{s^{n / v}+a_{1} s^{(n-1) / v}+\cdots+a_{n}}=0,
$$

which can be written as

$$
\frac{s^{n / v}+a_{1} s^{(n-1) / v}+\cdots+a_{n}}{s^{m / v}+b_{1} s^{(m-1) / v}+\cdots+b_{m}}=-k,
$$

then

$$
s^{(n-m) / v}\left(1+\frac{a_{1}-b_{1}}{s^{v}}+\cdots\right)=-k
$$


and for large $s$, it can be approximated as

$$
s^{(n-m) / v}\left(1+\frac{a_{1}-b_{1}}{s^{v}}\right) \sim-k .
$$

Taking both sides to power $v^{2} /(n-m)$ yields

$$
(-k)^{v^{2} /(n-m)} \sim S^{((n-m) / v) \times\left(v^{2} /(n-m)\right)}\left(1+\frac{a_{1}-b_{1}}{s^{v}}\right)^{v^{2} /(n-m)} .
$$

Now, using the fact that first-order Taylor's series expansion of $(1+x)^{r}$ around $x=0$ is $1+r x$, we have

$$
(-k)^{v^{2} /(n-m)} \sim s^{v}\left(1+\frac{v^{2}\left(a_{1}-b_{1}\right)}{s^{v}(n-m)}\right)=s^{v}+\frac{v^{2}\left(a_{1}-b_{1}\right)}{n-m} .
$$

Thus,

$$
\begin{aligned}
s^{v} & \sim(-1)^{v^{2} /(n-m)} k^{v^{2} /(n-m)}-\frac{\left(a_{1}-b_{1}\right) v^{2}}{n-m} \\
& \sim e^{j(2 h+1) \pi v^{2} /(n-m)} k^{v^{2} /(n-m)}-\frac{\left(a_{1}-b_{1}\right) v^{2}}{n-m} \\
& \sim e^{j(2 h+1) \pi v^{2} /(n-m)} k^{v^{2} /(n-m)}\left(1-\frac{e^{-j(2 h+1) \pi v^{2} /(n-m)}\left(a_{1}-b_{1}\right) v^{2}}{(n-m) k^{v^{2} /(n-m)}}\right) .
\end{aligned}
$$

Taking both sides to power $1 / v$ yields

$$
S \sim e^{j(2 h+1) \pi v /(n-m)} k^{v /(n-m)}\left(1-\frac{e^{-j(2 h+1) \pi v^{2} /(n-m)}\left(a_{1}-b_{1}\right) v^{2}}{(n-m) k^{v^{2} /(n-m)}}\right)^{1 / v} .
$$

Again using Taylor's series expansion,

$$
s \sim e^{j(2 h+1) \pi v /(n-m)} k^{v /(n-m)}\left(1-\frac{e^{-j(2 h+1) \pi v^{2} /(n-m)}\left(a_{1}-b_{1}\right) v}{(n-m) k^{v^{2} /(n-m)}}\right) .
$$

That can further be written as

$$
S \sim k^{v /(n-m)} e^{j(2 h+1) \pi v /(n-m)}-\frac{e^{-j(2 h+1) \pi\left(v^{2}-v\right) /(n-m)}\left(a_{1}-b_{1}\right) v}{(n-m) k^{\left(v^{2}-v\right) /(n-m)}} .
$$

In the above formula, the last term goes to zero for all $v>1$ as $k \rightarrow \infty$ provided that $\left(v^{2}-\right.$ $v) /(n-m)>0$. It goes to $\left(a_{1}-b_{1}\right) /(n-m)$ for $v=1$ which results in the well-known fact that the asymptotes intersect at $\left(a_{1}-b_{1}\right) /(n-m)$ for integer case [1]. 


\section{References}

[1] K. Ogata, Modern Control Engineering, Prentice-Hall, Upper Saddle River, NJ, USA, 4th edition, 2001.

[2] A. M. Krall, "The root locus method: a survey," SIAM Review, vol. 12, no. 1, pp. 64-72, 1970.

[3] A. M. Krall, "A closed expression for the root locus method," SIAM Journal on Applied Mathematics, vol. 11, no. 3, pp. 700-704, 1963.

[4] E. Bahar and M. Fitzwater, "Numerical technique to trace the loci of the complex roots of characteristic equations," SIAM Journal on Scientific and Statistical Computing, vol. 2, no. 4, pp. 389-403, 1981.

[5] C. I. Byrnes, D. S. Gilliam, and J. He, "Root-locus and boundary feedback design for a class of distributed parameter systems," SIAM Journal on Control and Optimization, vol. 32, no. 5, pp. 13641427, 1994.

[6] K. B. Oldham and J. Spanier, The Fractional Calculus: Theory and Applications of Differentiation and Integration to Arbitrary Order, Academic Press, New York, NY, USA, 1974.

[7] R. Hilfe, Ed., Applications of Fractional Calculus in Physics, World Scientific, River Edge, NJ, USA, 2000.

[8] M.-C. Viano, C. Deniau, and G. Oppenheim, "Continuous-time fractional ARMA processes," Statistics E Probability Letters, vol. 21, no. 4, pp. 323-336, 1994.

[9] Y.-Y. Tsao, B. Onaral, and H. H. Sun, "An algorithm for determining global parameters of minimumphase systems with fractional power spectra," IEEE Transactions on Instrumentation and Measurement, vol. 38, no. 3, pp. 723-729, 1989.

[10] T. Poinot and J.-C. Trigeassou, "Identification of fractional systems using an output-error technique," Nonlinear Dynamics, vol. 38, no. 1-4, pp. 133-154, 2004.

[11] B. M. Vinagre, V. Feliu, and J. J. Feliu, "Frequency domain identification of a flexible structure with Piezoelectric actuators using irrational transfer function models," in Proceedings of the 37th IEEE Conference on Decision and Control (CDC '98), vol. 2, pp. 1278-1280, Tampa, Fla USA, December 1998.

[12] A. Chauchois, D. Didier, A. Emmanuel, and D. Bruno, "Use of noninteger identification models for monitoring soil water content," Measurement Science and Technology, vol. 14, no. 6, pp. 868-874, 2003.

[13] I. Podlubny, "Fractional-order systems and $\mathrm{PI}^{\lambda} \mathrm{D}^{\mu}$-controllers," IEEE Transactions on Automatic Control, vol. 44, no. 1, pp. 208-214, 1999.

[14] D. Valério and J. Sá da Costa, "Tuning of fractional PID controllers with Ziegler-Nichols-type rules," Signal Processing, vol. 86, no. 10, pp. 2771-2784, 2006.

[15] H.-F. Raynaud and A. Zergaïnoh, "State-space representation for fractional order controllers," Automatica, vol. 36, no. 7, pp. 1017-1021, 2000.

[16] A. Oustaloup, B. Mathieu, and P. Lanusse, "The CRONE control of resonant plants: application to a flexible transmission," European Journal of Control, vol. 1, no. 2, pp. 113-121, 1995.

[17] A. Oustaloup, X. Moreau, and M. Nouillant, "The CRONE suspension," Control Engineering Practice, vol. 4, no. 8, pp. 1101-1108, 1996.

[18] K. S. Miller and B. Ross, An Introduction to the Fractional Calculus and Fractional Differential Equations, A Wiley-Interscience Publication, John Wiley \& Sons, New York, NY, USA, 1993.

[19] H. Beyer and S. Kempfle, "Definition of physically consistent damping laws with fractional derivatives," Zeitschrift für Angewandte Mathematik und Mechanik, vol. 75, no. 8, pp. 623-635, 1995.

[20] M. Ikeda and S. Takahashi, "Generalization of Routh's algorithm and stability criterion for non-integer integral system," Electronics and Communications in Japan, vol. 60, no. 2, pp. 41-50, 1977.

[21] W. R. LePage, Complex Variables and the Laplace Transform for Engineers, International Series in Pure and Applied Mathematics, McGraw-Hill, New York, NY, USA, 1961.

[22] G. A. Jones and D. Singerman, Complex Functions: An Algebraic and Geometric Viewpoint, Cambridge University Press, Cambridge, UK, 1987.

[23] B. Gross and E. P. Braga, Singularities of Linear System Functions, Elsevier, New York, NY, USA, 1961.

[24] D. Matignon, "Stability properties for generalized fractional differential systems," in Systèmes Différentiels Fractionnaires (Paris, 1998), vol. 5 of ESAIM Proceedings, pp. 145-158, SMAI, Paris, France, 1998.

[25] D. Matignon, "Stability results on fractional differential equations with applications to control processing," in Proceedings of the Computational Engineering in Systems Applications, CESA96 IMACSIEEE/SMC Multiconference, pp. 963-968, Lille, France, July 1996. 
[26] A. M. Eydgahi and M. Ghavamzadeh, "Complementary root locus revisited," IEEE Transactions on Education, vol. 44, no. 2, pp. 137-143, 2001.

[27] G. Berman and R. G. Stanton, "The asymptotes of the root locus," SIAM Review, vol. 5, no. 3, pp. 209-218, 1963.

[28] I. Podlubny, L. Dorcak, and I. Kostial, "On fractional derivatives, fractional order dynamic system and $\mathrm{PI}^{\lambda} \mathrm{D}^{\mu}$-controllers," in Proceedings of the 36th IEEE Conference on Decision and Control (CDC'97), vol. 5, pp. 4985-4990, San Diego, Calif, USA, December 1997.

[29] I. Podlubny, Fractional Differential Equations: An Introduction to Fractional Derivatives, Fractional Differential Equations, to Methods of Their Solution and Some of Their Applications, vol. 198 of Mathematics in Science and Engineering, Academic Press, San Diego, Calif, USA, 1999. 


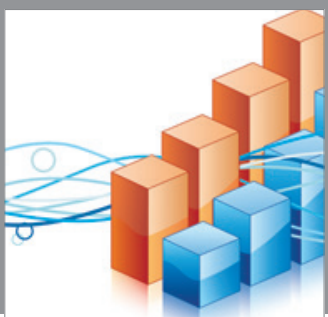

Advances in

Operations Research

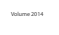

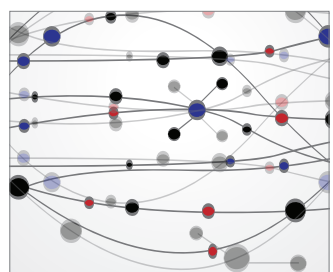

\section{The Scientific} World Journal
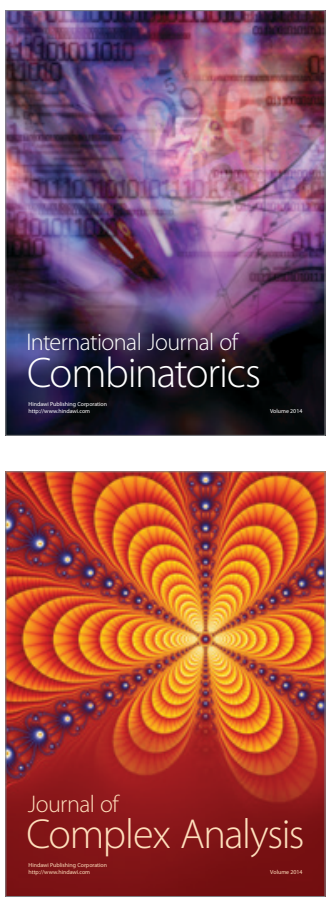

International Journal of

Mathematics and

Mathematical

Sciences
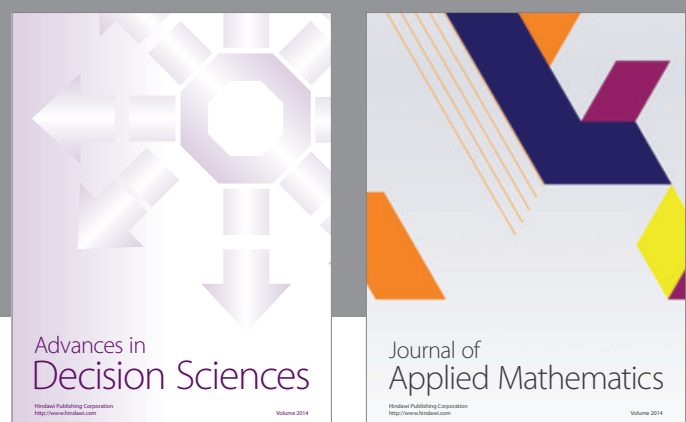

Journal of

Applied Mathematics
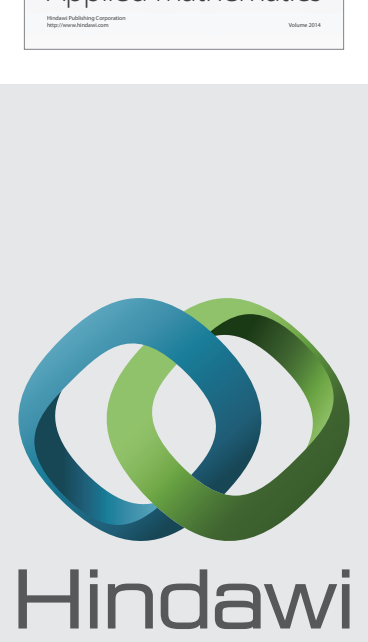

Submit your manuscripts at http://www.hindawi.com
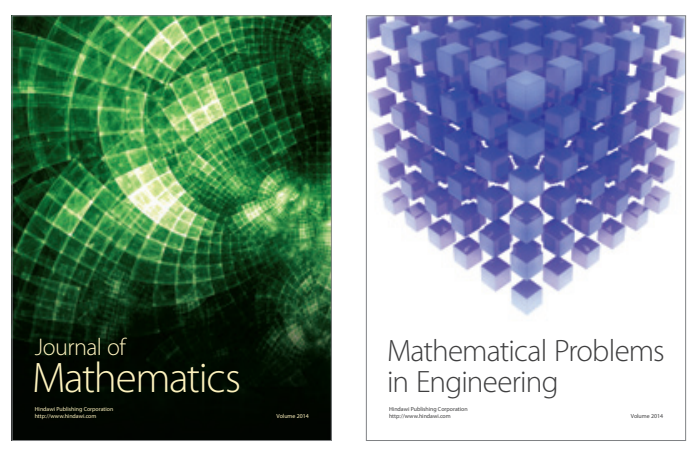

Mathematical Problems in Engineering
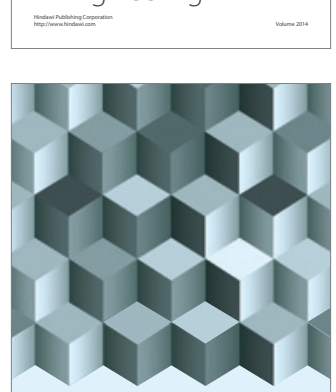

Journal of

Function Spaces
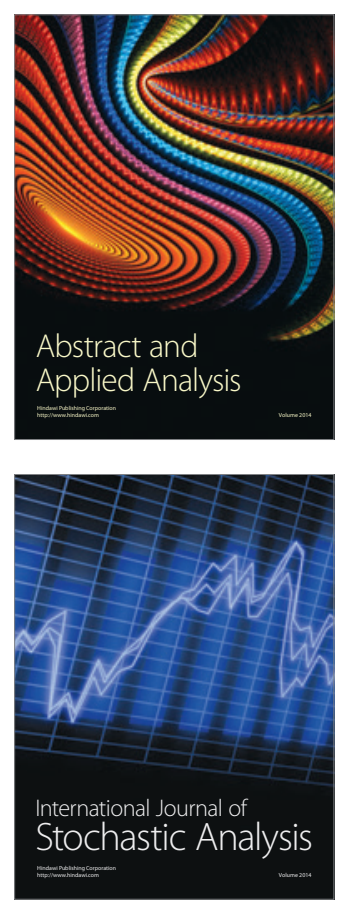

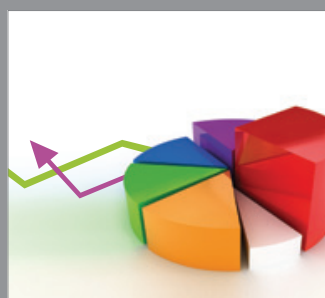

ournal of

Probability and Statistics

Promensencen
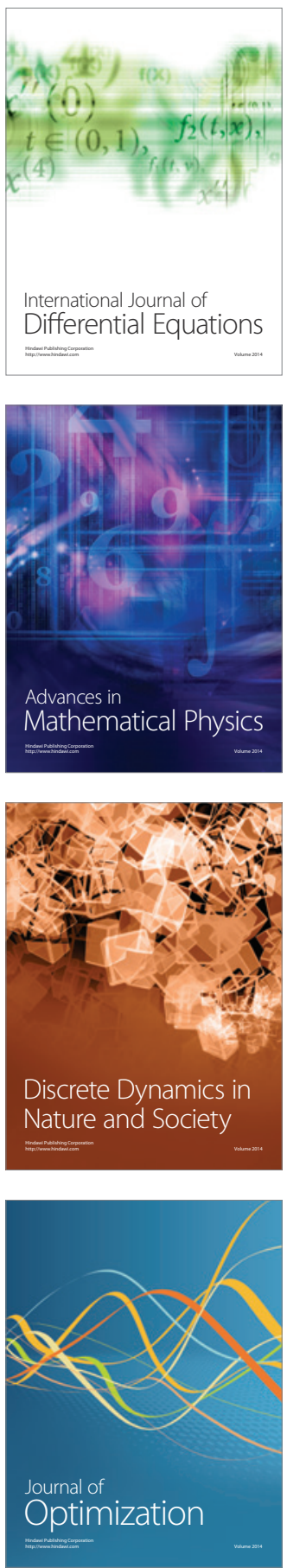